\title{
Building a Discourse-Tagged Corpus in the Framework of Rhetorical Structure Theory
}

\author{
Lynn Carlson \\ Department of Defense \\ Ft. George G. Meade \\ MD 20755 \\ lmcarlnord@aol.com
}

\author{
Daniel Marcu \\ Information Sciences Institute \\ University of S. California \\ Marina del Rey, CA 90292 \\ marcu@isi.edu
}

\author{
Mary Ellen Okurowski \\ Department of Defense \\ Ft. George G. Meade \\ MD 20755 \\ meokuro@romulus.ncsc.mil
}

\begin{abstract}
We describe our experience in developing a discourse-annotated corpus for community-wide use. Working in the framework of Rhetorical Structure Theory, we were able to create a large annotated resource with very high consistency, using a well-defined methodology and protocol. This resource is made publicly available through the Linguistic Data Consortium to enable researchers to develop empirically grounded, discourse-specific applications.
\end{abstract}

\section{Introduction}

The advent of large-scale collections of annotated data has marked a paradigm shift in the research community for natural language processing. These corpora, now also common in many languages, have accelerated development efforts and energized the community. Annotation ranges from broad characterization of document-level information, such as topic or relevance judgments (Voorhees and Harman, 1999; Wayne, 2000) to discrete analysis of a wide range of linguistic phenomena. However, rich theoretical approaches to discourse/text analysis (Van Dijk and Kintsch, 1983; Meyer, 1985; Grosz and Sidner, 1986; Mann and Thompson, 1988) have yet to be applied on a large scale. So far, the annotation of discourse structure of documents has been applied primarily to identifying topical segments (Hearst, 1997), inter-sentential relations (Nomoto and Matsumoto, 1999; Ts'ou et al., 2000), and hierarchical analyses of small corpora (Moser and Moore, 1995; Marcu et al., 1999).

In this paper, we recount our experience in developing a large resource with discourse-level annotation for NLP research. Our main goal in undertaking this effort was to create a reference corpus for community-wide use. Two essential considerations from the outset were that the corpus needed to be consistently annotated, and that it would be made publicly available through the Linguistic Data Consortium for a nominal fee to cover distribution costs. The paper describes the challenges we faced in building a corpus of this level of complexity and scope including selection of theoretical approach, annotation methodology, training, and quality assurance. The resulting corpus contains 385 documents of American English selected from the Penn Treebank (Marcus et al., 1993), annotated in the framework of Rhetorical Structure Theory. We believe this resource holds great promise as a rich new source of textlevel information to support multiple lines of research for language understanding applications.

\section{Framework}

Two principle goals underpin the creation of this discourse-tagged corpus: 1) The corpus should be grounded in a particular theoretical approach, and 2) it should be sufficiently large enough to offer potential for wide-scale use - including linguistic analysis, training of statistical models of discourse, and other computational linguistic applications. These goals necessitated a number of constraints to our approach. The theoretical framework had to be practical and repeatable over a large set of documents in a reasonable amount of time, with a significant level of consistency across annotators. Thus, our 
approach contributes to the community quite differently from detailed analyses of specific discourse phenomena in depth, such as anaphoric relations (Garside et al., 1997) or style types (Leech et al., 1997); analysis of a single text from multiple perspectives (Mann and Thompson, 1992); or illustrations of a theoretical model on a single representative text (Britton and Black, 1985; Van Dijk and Kintsch, 1983).

Our annotation work is grounded in the Rhetorical Structure Theory (RST) framework (Mann and Thompson, 1988). We decided to use RST for three reasons:

- It is a framework that yields rich annotations that uniformly capture intentional, semantic, and textual features that are specific to a given text.

- Previous research on annotating texts with rhetorical structure trees (Marcu et al., 1999) has shown that texts can be annotated by multiple judges at relatively high levels of agreement. We aimed to produce annotation protocols that would yield even higher agreement figures.

- Previous research has shown that RST trees can play a crucial role in building natural language generation systems (Hovy, 1993; Moore and Paris, 1993; Moore, 1995) and text summarization systems (Marcu, 2000); can be used to increase the naturalness of machine translation outputs (Marcu et al. 2000); and can be used to build essayscoring systems that provide students with discourse-based feedback (Burstein et al., 2001). We suspect that RST trees can be exploited successfully in the context of other applications as well.

In the RST framework, the discourse structure of a text can be represented as a tree defined in terms of four aspects:

- The leaves of the tree correspond to text fragments that represent the minimal units of the discourse, called elementary discourse units

- The internal nodes of the tree correspond to contiguous text spans

- Each node is characterized by its nuclearity - a nucleus indicates a more essential unit of information, while a satellite indicates a supporting or background unit of information.

- Each node is characterized by a rhetorical relation that holds between two or more non-overlapping, adjacent text spans. Relations can be of intentional, semantic, or textual nature.

Below, we describe the protocol that we used to build consistent RST annotations.

\subsection{Segmenting Texts into Units}

The first step in characterizing the discourse structure of a text in our protocol is to determine the elementary discourse units (EDUs), which are the minimal building blocks of a discourse tree. Mann and Thompson (1988, p. 244) state that "RST provides a general way to describe the relations among clauses in a text, whether or not they are grammatically or lexically signalled." Yet, applying this intuitive notion to the task of producing a large, consistently annotated corpus is extremely difficult, because the boundary between discourse and syntax can be very blurry. The examples below, which range from two distinct sentences to a single clause, all convey essentially the same meaning, packaged in different ways:

1. [Xerox Corp.'s third-quarter net income grew $6.2 \%$ on $7.3 \%$ higher revenue.] [This earned mixed reviews from Wall Street analysts.]

2. [Xerox Corp's third-quarter net income grew $6.2 \%$ on $7.3 \%$ higher revenue,] [which earned mixed reviews from Wall Street analysts.]

3. [Xerox Corp's third-quarter net income grew $6.2 \%$ on $7.3 \%$ higher revenue,] [earning mixed reviews from Wall Street analysts.]

4. [The $6.2 \%$ growth of Xerox Corp.'s thirdquarter net income on $7.3 \%$ higher revenue earned mixed reviews from Wall Street analysts.]

In Example 1, there is a consequential relation between the first and second sentences. Ideally, we would like to capture that kind of rhetorical information regardless of the syntactic form in which it is conveyed. However, as examples 2-4 illustrate, separating rhetorical 
from syntactic analysis is not always easy. It is inevitable that any decision on how to bracket elementary discourse units necessarily involves some compromises.

Reseachers in the field have proposed a number of competing hypotheses about what constitutes an elementary discourse unit. While some take the elementary units to be clauses (Grimes, 1975; Givon, 1983; Longacre, 1983), others take them to be prosodic units (Hirschberg and Litman, 1993), turns of talk (Sacks, 1974), sentences (Polanyi, 1988), intentionally defined discourse segments (Grosz and Sidner, 1986), or the "contextually indexed representation of information conveyed by a semiotic gesture, asserting a single state of affairs or partial state of affairs in a discourse world," (Polanyi, 1996, p.5). Regardless of their theoretical stance, all agree that the elementary discourse units are non-overlapping spans of text.

Our goal was to find a balance between granularity of tagging and ability to identify units consistently on a large scale. In the end, we chose the clause as the elementary unit of discourse, using lexical and syntactic clues to help determine boundaries:

5. [Although Mr. Freeman is retiring,] [he will continue to work as a consultant for American Express on a project basis. $]_{\text {wsj_1317 }}$

6. [Bond Corp., a brewing, property, media and resources company, is selling many of its assets] [to reduce its debts.] $]_{\text {wsj_0630 }}$

However, clauses that are subjects, objects, or complements of a main verb are not treated as EDUs:

7. [Making computers smaller often means sacrificing memory. $]_{\mathrm{wsj} \_2387}$

8. [Insurers could see claims totaling nearly \$1 billion from the San Francisco earthquake. $]_{\text {wsj_0675 }}$

Relative clauses, nominal postmodifiers, or clauses that break up other legitimate EDUs, are treated as embedded discourse units:

9. [The results underscore Sears's difficulties] [in implementing the "everyday low pricing” strategy... $]_{\mathrm{wsj} \_1105}$

10. [The Bush Administration,] [trying to blunt growing demands from Western Europe for a relaxation of controls on exports to the

Soviet bloc,] [is questioning... $]_{\mathrm{wsj} \_2326}$

Finally, a small number of phrasal EDUs are allowed, provided that the phrase begins with a strong discourse marker, such as because, in spite of, as a result of, according to. We opted for consistency in segmenting, sacrificing some potentially discourse-relevant phrases in the process.

\subsection{Building up the Discourse Structure}

Once the elementary units of discourse have been determined, adjacent spans are linked together via rhetorical relations creating a hierarchical structure. Relations may be mononuclear or multinuclear. Mononuclear relations hold between two spans and reflect the situation in which one span, the nucleus, is more salient to the discourse structure, while the other span, the satellite, represents supporting information. Multinuclear relations hold among two or more spans of equal weight in the discourse structure. A total of 53 mononuclear and 25 multinuclear relations were used for the tagging of the RST Corpus. The final inventory of rhetorical relations is data driven, and is based on extensive analysis of the corpus. Although this inventory is highly detailed, annotators strongly preferred keeping a higher level of granularity in the selections available to them during the tagging process. More extensive analysis of the final tagged corpus will demonstrate the extent to which individual relations that are similar in semantic content were distinguished consistently during the tagging process.

The 78 relations used in annotating the corpus can be partitioned into 16 classes that share some type of rhetorical meaning: Attribution, Background, Cause, Comparison, Condition, Contrast, Elaboration, Enablement, Evaluation, Explanation, Joint, Manner-Means, Topic-Comment, Summary, Temporal, TopicChange. For example, the class Explanation includes the relations evidence, explanationargumentative, and reason, while TopicComment includes problem-solution, questionanswer, statement-response, topic-comment, and comment-topic. In addition, three relations are used to impose structure on the tree: textualorganization, span, and same-unit (used to link 
parts of units separated by embedded units or spans).

\section{Discourse Annotation Task}

Our methodology for annotating the RST Corpus builds on prior corpus work in the Rhetorical Structure Theory framework by Marcu et al. (1999). Because the goal of this effort was to build a high-quality, consistently annotated reference corpus, the task required that we employ people as annotators whose primary professional experience was in the area of language analysis and reporting, provide extensive annotator training, and specify a rigorous set of annotation guidelines.

\subsection{Annotator Profile and Training}

The annotators hired to build the corpus were all professional language analysts with prior experience in other types of data annotation. They underwent extensive hands-on training, which took place roughly in three phases. During the orientation phase, the annotators were introduced to the principles of Rhetorical Structure Theory and the discourse-tagging tool used for the project (Marcu et al., 1999). The tool enables an annotator to segment a text into units, and then build up a hierarchical structure of the discourse. In this stage of the training, the focus was on segmenting hard copy texts into EDUs, and learning the mechanics of the tool.

In the second phase, annotators began to explore interpretations of discourse structure, by independently tagging a short document, based on an initial set of tagging guidelines, and then meeting as a group to compare results. The initial focus was on resolving segmentation differences, but over time this shifted to addressing issues of relations and nuclearity. These exploratory sessions led to enhancements in the tagging guidelines. To reinforce new rules, annotators re-tagged the document. During this process, we regularly tracked interannotator agreement (see Section 4.2). In the final phase, the annotation team concentrated on ways to reduce differences by adopting some heuristics for handling higher levels of the discourse structure. Wiebe et al. (1999) present a method for automatically formulating a single best tag when multiple judges disagree on selecting between binary features. Because our annotators had to select among multiple choices at each stage of the discourse annotation process, and because decisions made at one stage influenced the decisions made during subsequent stages, we could not apply Wiebe et al.'s method. Our methodology for determining the "best" guidelines was much more of a consensus-building process, taking into consideration multiple factors at each step. The final tagging manual, over 80 pages in length, contains extensive examples from the corpus to illustrate text segmentation, nuclearity, selection of relations, and discourse cues. The manual can be downloaded from the following web site: http://www.isi.edu/ marcu/discourse.

The actual tagging of the corpus progressed in three developmental phases. During the initial phase of about four months, the team created a preliminary corpus of 100 tagged documents. This was followed by a one-month reassessment phase, during which we measured consistency across the group on a select set of documents, and refined the annotation rules. At this point, we decided to proceed by pre-segmenting all of the texts on hard copy, to ensure a higher overall quality to the final corpus. Each text was presegmented by two annotators; discrepancies were resolved by the author of the tagging guidelines. In the final phase (about six months) all 100 documents were re-tagged with the new approach and guidelines. The remainder of the corpus was tagged in this manner.

\subsection{Tagging Strategies}

Annotators developed different strategies for analyzing a document and building up the corresponding discourse tree. There were two basic orientations for document analysis - hard copy or graphical visualization with the tool. Hard copy analysis ranged from jotting of notes in the margins to marking up the document into discourse segments. Those who preferred a graphical orientation performed their analysis simultaneously with building the discourse structure, and were more likely to build the discourse tree in chunks, rather than incrementally.

We observed a variety of annotation styles for the actual building of a discourse tree. Two of the more representative styles are illustrated below.

1. The annotator segments the text one unit at a time, then incrementally builds up the 
discourse tree by immediately attaching the current node to a previous node. When building the tree in this fashion, the annotator must anticipate the upcoming discourse structure, possibly for a large span. Yet, often an appropriate choice of relation for an unseen segment may not be obvious from the current (rightmost) unit that needs to be attached. That is why annotators typically used this approach on short documents, but resorted to other strategies for longer documents.

2. The annotator segments multiple units at a time, then builds discourse sub-trees for each sentence. Adjacent sentences are then linked, and larger sub-trees begin to emerge. The final tree is produced by linking major chunks of the discourse
Corp. ${ }^{18}$ [This is in part because of the effect ${ }^{19}$ [of having to average the number of shares outstanding, $]^{20}$ [she said. ${ }^{21}$ [In addition, $]^{22}$ [Mrs. Lidgerwood said, $]^{23}$ [Norfolk is likely to draw down its cash initially $]^{24}$ [to finance the purchases] $]^{25}$ [and thus forfeit some interest income. ${ }^{26}{ }_{\text {wsj_1111 }}$

The discourse sub-tree for this text fragment is given in Figure 1. Using Style 1 the annotator, upon segmenting unit [17], must anticipate the upcoming example relation, which spans units [17-26]. However, even if the annotator selects an incorrect relation at that point, the tool allows great flexibility in changing the structure of the tree later on.

Using Style 2, the annotator segments each sentence, and builds up corresponding sub-trees for spans [16], [17-18], [19-21] and [22-26]. The

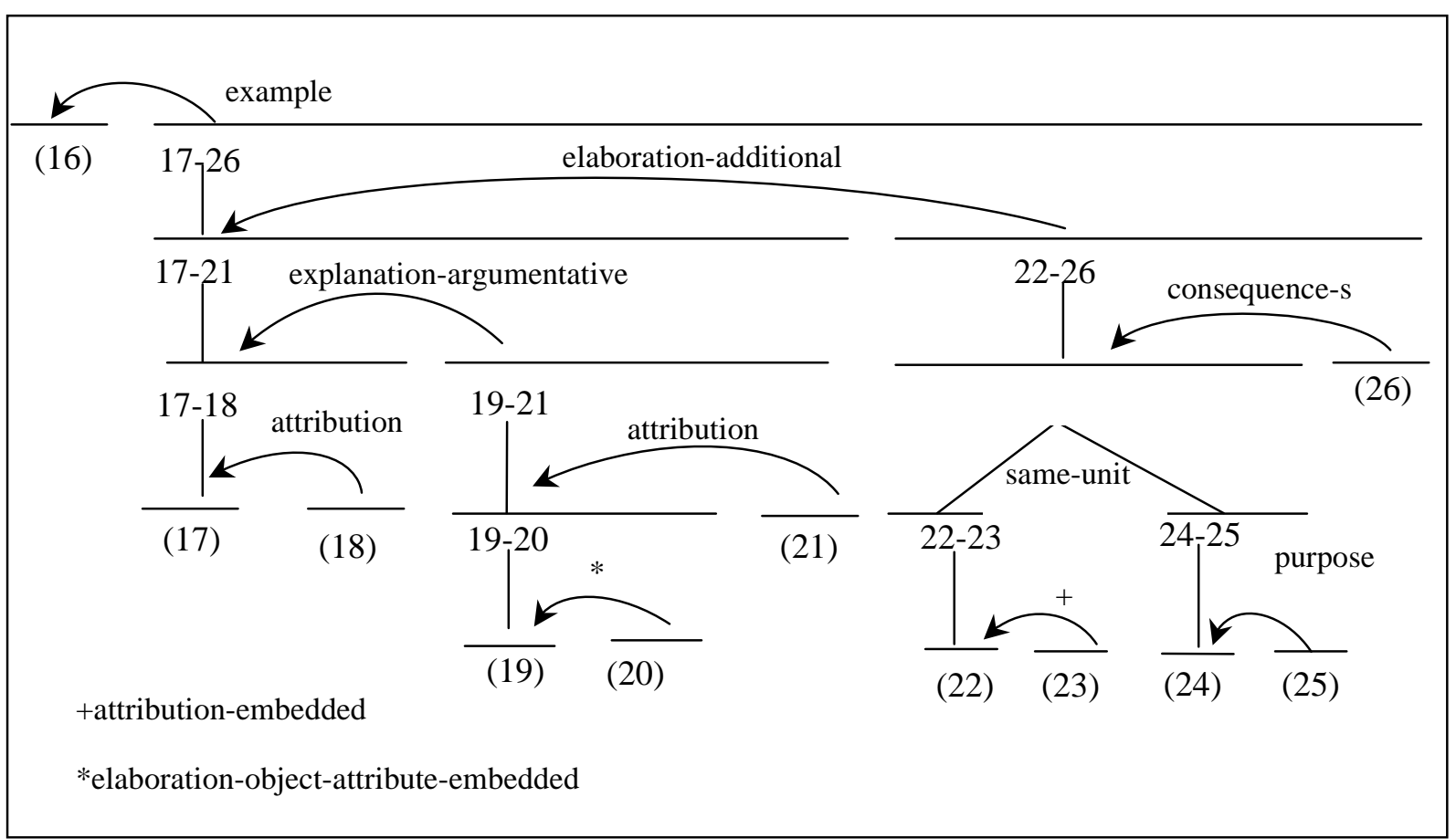

Figure 1: Discourse sub-tree for multiple sentences

structure. This strategy allows the annotator to see the emerging discourse structure more globally; thus, it was the preferred approach for longer documents.

Consider the text fragment below, consisting of four sentences, and 11 EDUs:

[Still, analysts don't expect the buy-back to significantly affect per-share earnings in the short term. $]^{16}[\text { The impact won't be that great, }]^{17}$ [said Graeme Lidgerwood of First Boston second and third sub-trees are then linked via an explanation-argumentative relation, after which, the fourth sub-tree is linked via an elaborationadditional relation. The resulting span [17-26] is finally attached to node [16] as an example satellite.

\section{Quality Assurance}

A number of steps were taken to ensure the quality of the final discourse corpus. These 
Table 1: Inter-annotator agreement - periodic results for three taggers

\begin{tabular}{|c|c|c|c|c|c|c|c|}
\hline Taggers & Units & Spans & Nuclearity & Relations & $\begin{array}{c}\text { Fewer- } \\
\text { Relations }\end{array}$ & $\begin{array}{c}\text { No. of } \\
\text { Docs }\end{array}$ & $\begin{array}{c}\text { Avg. No. } \\
\text { EDUs }\end{array}$ \\
\hline $\begin{array}{c}\mathbf{A}, \mathbf{B}, \mathbf{E} \\
\text { (Apr 00) }\end{array}$ & $\mathbf{0 . 8 7 4 4 0 7}$ & $\mathbf{0 . 7 7 2 1 4 7}$ & $\mathbf{0 . 7 0 5 3 3 0}$ & $\mathbf{0 . 6 0 1 6 7 3}$ & $\mathbf{0 . 6 4 4 8 5 1}$ & $\mathbf{4}$ & $\mathbf{1 2 8 . 7 5 0 0 0 0}$ \\
\hline $\begin{array}{c}\mathrm{A}, \mathrm{B}, \mathrm{E} \\
\text { (Jun 00) }\end{array}$ & 0.952721 & 0.844141 & 0.782589 & 0.708932 & 0.739616 & 5 & 38.400002 \\
\hline $\begin{array}{c}\mathrm{A}, \mathrm{E} \\
(\text { Nov 00) }\end{array}$ & 0.984471 & 0.904707 & 0.835040 & 0.755486 & 0.784435 & 6 & 57.666668 \\
\hline $\begin{array}{c}\text { B, E } \\
(\text { Nov 00) }\end{array}$ & 0.960384 & 0.890481 & 0.848976 & 0.782327 & 0.806389 & 7 & 88.285713 \\
\hline $\begin{array}{c}\text { A, B } \\
\text { (Nov 00) }\end{array}$ & 1.000000 & 0.929157 & 0.882437 & 0.792134 & 0.822910 & 5 & 58.200001 \\
\hline $\begin{array}{c}\mathbf{A}, \mathbf{B}, \mathbf{E} \\
\text { (Jan 01) }\end{array}$ & $\mathbf{0 . 9 7 1 6 1 3}$ & $\mathbf{0 . 8 9 9 9 7 1}$ & $\mathbf{0 . 8 5 5 8 6 7}$ & $\mathbf{0 . 7 5 5 5 3 9}$ & $\mathbf{0 . 7 8 2 3 1 2}$ & $\mathbf{5}$ & $\mathbf{6 8 . 5 9 9 9 9 8}$ \\
\hline
\end{tabular}

involved two types of tasks: checking the validity of the trees and tracking inter-annotator consistency.

\subsection{Tree Validation Procedures}

Annotators reviewed each tree for syntactic and semantic validity. Syntactic checking involved ensuring that the tree had a single root node and comparing the tree to the document to check for missing sentences or fragments from the end of the text. Semantic checking involved reviewing nuclearity assignments, as well as choice of relation and level of attachment in the tree. All trees were checked with a discourse parser and tree traversal program which often identified errors undetected by the manual validation process. In the end, all of the trees worked successfully with these programs.

\subsection{Measuring Consistency}

We tracked inter-annotator agreement during each phase of the project, using a method developed by Marcu et al. (1999) for computing kappa statistics over hierarchical structures. The kappa coefficient (Siegel and Castellan, 1988) has been used extensively in previous empirical studies of discourse (Carletta et al., 1997; Flammia and Zue, 1995; Passonneau and Litman, 1997). It measures pairwise agreement among a set of coders who make category judgments, correcting for chance expected agreement. The method described in Marcu et al. (1999) maps hierarchical structures into sets of units that are labeled with categorial judgments. The strengths and shortcomings of the approach are also discussed in detail there. Researchers in content analysis (Krippendorff, 1980) suggest that values of kappa $>0.8$ reflect very high agreement, while values between 0.6 and 0.8 reflect good agreement.

Table 1 shows average kappa statistics reflecting the agreement of three annotators at various stages of the tasks on selected documents. Different sets of documents were chosen for each stage, with no overlap in documents. The statistics measure annotation reliability at four levels: elementary discourse units, hierarchical spans, hierarchical nuclearity and hierarchical relation assignments.

At the unit level, the initial (April 00) scores and final (January 01) scores represent agreement on blind segmentation, and are shown in boldface. The interim June and November scores represent agreement on hard copy pre-segmented texts. Notice that even with pre-segmenting, the agreement on units is not $100 \%$ perfect, because of human errors that occur in segmenting with the tool. As Table 1 shows, all levels demonstrate a marked improvement from April to November (when the final corpus was completed), ranging from about 0.77 to 0.92 at the span level, from 0.70 to 0.88 at the nuclearity level, and from 0.60 to 0.79 at the relation level. In particular, when relations are combined into the 16 rhetoricallyrelated classes discussed in Section 2.2, the November results of the annotation process are extremely good. The Fewer-Relations column shows the improvement in scores on assigning 
Table 2: Inter-annotator agreement - final results fox six taggers

\begin{tabular}{|l|l|l|l|r|r|r|r|}
\hline Taggers & Units & Spans & Nuclearity & Relations & $\begin{array}{l}\text { Fewer- } \\
\text { Relations }\end{array}$ & $\begin{array}{l}\text { No. of } \\
\text { Docs }\end{array}$ & $\begin{array}{l}\text { Avg. No. } \\
\text { EDUs }\end{array}$ \\
\hline B, E & 0.960384 & 0.890481 & 0.848976 & 0.782327 & 0.806389 & 7 & 88.285713 \\
\hline A, E & 0.984471 & 0.904707 & 0.835040 & 0.755486 & 0.784435 & 6 & 57.666668 \\
\hline A, B & 1.000000 & 0.929157 & 0.882437 & 0.792134 & 0.822910 & 5 & 58.200001 \\
\hline A, C & 0.950962 & 0.840187 & 0.782688 & 0.676564 & 0.711109 & 4 & 116.500000 \\
\hline A, F & 0.952342 & 0.777553 & 0.694634 & 0.597302 & 0.624908 & 4 & 26.500000 \\
\hline A, D & 1.000000 & 0.868280 & 0.801544 & 0.720692 & 0.769894 & 4 & 23.250000 \\
\hline
\end{tabular}

relations when they are grouped in this manner, with November results ranging from 0.78 to 0.82. In order to see how much of the improvement had to do with pre-segmenting, we asked the same three annotators to annotate five previously unseen documents in January, without reference to a pre-segmented document. The results of this experiment are given in the last row of Table 1, and they reflect only a small overall decline in performance from the November results. These scores reflect very strong agreement and represent a significant improvement over previously reported results on annotating multiple texts in the RST framework (Marcu et al., 1999).

Table 2 reports final results for all pairs of taggers who double-annotated four or more documents, representing 30 out of the 53 documents that were double-tagged. Results are based on pre-segmented documents.

Our team was able to reach a significant level of consistency, even though they faced a number of challenges which reflect differences in the agreement scores at the various levels. While operating under the constraints typical of any theoretical approach in an applied environment, the annotators faced a task in which the complexity increased as support from the guidelines tended to decrease. Thus, while rules for segmenting were fairly precise, annotators relied on heuristics requiring more human judgment to assign relations and nuclearity. Another factor is that the cognitive challenge of the task increases as the tree takes shape. It is relatively straightforward for the annotator to make a decision on assignment of nuclearity and relation at the inter-clausal level, but this becomes more complex at the intersentential level, and extremely difficult when linking large segments.
This tension between task complexity and guideline under-specification resulted from the practical application of a theoretical model on a broad scale. While other discourse theoretical approaches posit distinctly different treatments for various levels of the discourse (Van Dijk and Kintsch, 1983; Meyer, 1985), RST relies on a standard methodology to analyze the document at all levels. The RST relation set is rich and the concept of nuclearity, somewhat interpretive. This gave our annotators more leeway in interpreting the higher levels of the discourse structure, thus introducing some stylistic differences, which may prove an interesting avenue of future research.

\section{Corpus Details}

The RST Corpus consists of 385 Wall Street Journal articles from the Penn Treebank, representing over 176,000 words of text. In order to measure inter-annotator consistency, 53 of the documents $(13.8 \%)$ were double-tagged. The documents range in size from 31 to 2124 words, with an average of 458.14 words per document. The final tagged corpus contains 21,789 EDUs with an average of 56.59 EDUs per document. The average number of words per EDU is 8.1.

The articles range over a variety of topics, including financial reports, general interest stories, business-related news, cultural reviews, editorials, and letters to the editor. In selecting these documents, we partnered with the Linguistic Data Consortium to select Penn Treebank texts for which the syntactic bracketing was known to be of high caliber. Thus, the RST Corpus provides an additional level of linguistic annotation to supplement existing annotated resources. 
For details on obtaining the corpus, annotation software, tagging guidelines, and related documentation and resources, see: http://www.isi.edu/ marcu/discourse.

\section{Discussion}

A growing number of groups have developed or are developing discourse-annotated corpora for text. These can be characterized both in terms of the kinds of features annotated as well as by the scope of the annotation. Features may include specific discourse cues or markers, coreference links, identification of rhetorical relations, etc. The scope of the annotation refers to the levels of analysis within the document, and can be characterized as follows:

- sentential: annotation of features at the intra-sentential or inter-sentential level, at a single level of depth (Sundheim, 1995; Tsou et al., 2000; Nomoto and Matsumoto, 1999; Rebeyrolle, 2000).

- hierarchical: annotation of features at multiple levels, building upon lower levels of analysis at the clause or sentence level (Moser and Moore, 1995; Marcu, et al. 1999)

- document-level: broad characterization of document structure such as identification of topical segments (Hearst, 1997), linking of large text segments via specific relations (Ferrari, 1998; Rebeyrolle, 2000), or defining text objects with a text architecture (Pery-Woodley and Rebeyrolle, 1998).

Developing corpora with these kinds of rich annotation is a labor-intensive effort. Building the RST Corpus involved more than a dozen people on a full or part-time basis over a oneyear time frame (Jan. - Dec. 2000). Annotation of a single document could take anywhere from 30 minutes to several hours, depending on the length and topic. Re-tagging of a large number of documents after major enhancements to the annotation guidelines was also time consuming. In addition, limitations of the theoretical approach became more apparent over time. Because the RST theory does not differentiate between different levels of the tree structure, a fairly fine-grained set of relations operates between EDUs and EDU clusters at the macrolevel. The procedural knowledge available at the
EDU level is likely to need further refinement for higher-level text spans along the lines of other work which posits a few macro-level relations for text segments, such as Ferrari (1998) or Meyer (1985). Moreover, using the RST approach, the resultant tree structure, like a traditional outline, imposed constraints that other discourse representations (e.g., graph) would not. In combination with the tree structure, the concept of nuclearity also guided an annotator to capture one of a number of possible stylistic interpretations. We ourselves are eager to explore these aspects of the RST, and expect new insights to appear through analysis of the corpus.

We anticipate that the RST Corpus will be multifunctional and support a wide range of language engineering applications. The added value of multiple layers of overt linguistic phenomena enhancing the Penn Treebank information can be exploited to advance the study of discourse, to enhance language technologies such as text summarization, machine translation or information retrieval, or to be a testbed for new and creative natural language processing techniques.

\section{References}

Bruce Britton and John Black. 1985. Understanding Expository Text. Hillsdale, NJ: Lawrence Erlbaum Associates.

Jill Burstein, Daniel Marcu, Slava Andreyev, and Martin Chodorow. 2001. Towards automatic identification of discourse elements in essays. In Proceedings of the $39^{\text {th }}$ Annual Meeting of the Association for Computational Linguistics, Toulouse, France.

Jean Carletta, Amy Isard, Stephen Isard, Jacqueline Kowtko, Gwyneth Doherty-Sneddon, and Anne Anderson. 1997. The reliability of a dialogue structure coding scheme. Computational Linguistics 23(1): 13-32.

Giacomo Ferrari. 1998. Preliminary steps toward the creation of a discourse and text resource. In Proceedings of the First International Conference on Language Resources and Evaluation (LREC 1998), Granada, Spain, 999-1001.

Giovanni Flammia and Victor Zue. 1995. Empirical evaluation of human performance and agreement in parsing discourse constituents in 
spoken dialogue. In Proceedings of the $4^{\text {th }}$ European Conference on Speech Communication and Technology, Madrid, Spain, vol. 3, 1965-1968.

Roger Garside, Steve Fligelstone and Simon Botley. 1997. Discourse Annotation: Anaphoric Relations in Corpora. In Corpus annotation: Linguistic information from computer text corpora, edited by R. Garside, G. Leech, and T. McEnery. London: Longman, 66-84.

Talmy Givon. 1983. Topic continuity in discourse. In Topic Continuity in Discourse: a Quantitative Cross-Language Study. Amsterdam/Philadelphia: John Benjamins, 1-41.

Joseph Evans Grimes. 1975. The Thread of Discourse. The Hague, Paris: Mouton.

Barbara Grosz and Candice Sidner. 1986. Attentions, intentions, and the structure of discourse. Computational Linguistics, 12(3): 175-204.

Marti Hearst. 1997. TextTiling: Segmenting text into multi-paragraph subtopic passages. Computational Linguistics 23(1): 33-64.

Julia Hirschberg and Diane Litman. 1993. Empirical studies on the disambiguation of cue phrases. Computational Linguistics 19(3): 501530.

Eduard Hovy. 1993. Automated discourse generation using discourse structure relations. Artificial Intelligence 63(1-2): 341-386.

Klaus Krippendorff. 1980. Content Analysis: An Introduction to its Methodology. Beverly Hills, CA: Sage Publications.

Geoffrey Leech, Tony McEnery, and Martin Wynne. 1997. Further levels of annotation. In Corpus Annotation: Linguistic Information from Computer Text Corpora, edited by R. Garside, G. Leech, and T. McEnery. London: Longman, 85-101.

Robert Longacre. 1983. The Grammar of Discourse. New York: Plenum Press.

William Mann and Sandra Thompson. 1988. Rhetorical structure theory. Toward a functional theory of text organization. Text, 8(3): 243-281.

William Mann and Sandra Thompson, eds. 1992. Discourse Description: Diverse Linguistic Analyses of a Fund-raising Text. Amsterdam/Philadelphia: John Benjamins.

Daniel Marcu. 2000. The Theory and Practice of Discourse Parsing and Summarization. Cambridge, MA: The MIT Press.
Daniel Marcu, Estibaliz Amorrortu, and Magdelena Romera. 1999. Experiments in constructing a corpus of discourse trees. In Proceedings of the ACL Workshop on Standards and Tools for Discourse Tagging, College Park, MD, 48-57.

Daniel Marcu, Lynn Carlson, and Maki Watanabe. 2000. The automatic translation of discourse structures. Proceedings of the First Annual Meeting of the North American Chapter of the Association for Computational Linguistics, Seattle, WA, 9-17.

Mitchell Marcus, Beatrice Santorini, and Mary Ann Marcinkiewicz. 1993. Building a large annotated corpus of English: the Penn Treebank, Computational Linguistics 19(2), 313-330.

Bonnie Meyer. 1985. Prose Analysis: Purposes, Procedures, and Problems. In Understanding Expository Text, edited by B. Britton and J. Black. Hillsdale, NJ: Lawrence Erlbaum Associates, 11-64.

Johanna Moore. 1995. Participating in Explanatory Dialogues: Interpreting and Responding to Questions in Context. Cambridge, MA: MIT Press.

Johanna Moore and Cecile Paris. 1993. Planning text for advisory dialogues: capturing intentional and rhetorical information. Computational Linguistics 19(4): 651-694.

Megan Moser and Johanna Moore. 1995. Investigating cue selection and placement in tutorial discourse. Proceedings of the $33^{\text {rd }}$ Annual Meeting of the Association for Computational Linguistics, Cambridge, MA, 130-135.

Tadashi Nomoto and Yuji Matsumoto. 1999. Learning discourse relations with active data selection. In Proceedings of the Joint SIGDAT Conference on Empirical Methods in Natural Language Processing and Very Large Corpora, College Park, MD, 158-167.

Rebecca Passonneau and Diane Litman. 1997. Discourse segmentation by human and automatic means. Computational Linguistics 23(1): 103-140.

Marie-Paule Pery-Woodley and Josette Rebeyrolle. 1998. Domain and genre in sublanguage text: definitional microtexts in three corpora. In Proceedings of the First International Conference on Language Resources and Evaluation (LREC-1998), Granada, Spain, 987-992. 
Livia Polanyi. 1988. A formal model of the structure of discourse. Journal of Pragmatics 12: 601-638.

Livia Polanyi. 1996. The linguistic structure of discourse. Center for the Study of Language and Information. CSLI-96-200.

Josette Rebeyrolle. 2000. Utilisation de contextes définitoires pour l'acquisition de connaissances à partir de textes. In Actes Journées Francophones d'Ingénierie de la Connaissance (IC'2000), Toulouse, IRIT, 105114.

Harvey Sacks, Emmanuel Schegloff, and Gail Jefferson. 1974. A simple systematics for the organization of turntaking in conversation. Language 50: 696-735.

Sidney Siegal and N.J. Castellan. 1988. Nonparametric Statistics for the Behavioral Sciences. New York: McGraw-Hill.

Beth Sundheim. 1995. Overview of results of the MUC-6 evaluation. In Proceedings of the Sixth Message Understanding Conference (MUC-6), Columbia, MD, 13-31.

Benjamin K. T'sou, Tom B.Y. Lai, Samuel W.K. Chan, Weijun Gao, and Xuegang Zhan. 2000. Enhancement of Chinese discourse marker tagger with C.4.5. In Proceedings of the Second Chinese Language Processing Workshop, Hong Kong, 38-45.

Teun A. Van Dijk and Walter Kintsch. 1983. Strategies of Discourse Comprehension. New York: Academic Press.

Ellen Voorhees and Donna Harman. 1999. The Eighth Text Retrieval Conference (TREC8). NIST Special Publication 500-246.

Charles Wayne. 2000. Multilingual topic detection and tracking: successful research enabled by corpora and evaluation. In Proceedings of the Second International Conference on Language Resources and Evaluation (LREC-2000), Athens, Greece, 1487-1493.

Janyce Wiebe, Rebecca Bruce, and Thomas O'Hara. 1999. Development and use of a goldstandard data set for subjectivity classifications. In Proceedings of the $37^{\text {th }}$ Annual Meeting of the Association for Computational Linguistics. College Park, MD, 246-253. 